\title{
TSOs and DSOs Collaboration: The Need for Data Exchange
}

\author{
Rui Alves ${ }^{1}$, Francisco Reis ${ }^{1}$, Caihao Liang ${ }^{1,2}$
}

\begin{abstract}
This paper presents common challenges facing Transmission Systems Operators (TSOs) and Distribution System Operators (DSOs), as well as the correlated need for a stronger cooperation between them in an environment of unbundled power systems. It addresses different operational and planning areas where the interaction between TSOs and DSOs is presently scarce, showing, through explanatory case-studies, the benefits of an improved and further structured data exchange among grid operators.

Outcomes demonstrate that TSO and DSO information exchange allows improved levels of renewable energy sources penetration, as well as an important contribute to help system operators enhancing both short and long-term system security.
\end{abstract}

\section{Keywords}

TSO-DSO Cooperation Challenges, Data Exchange, System Observability, Congestion Management, Voltage Control.

\author{
${ }^{1}$ R\&D Nester \\ Centro de Investigação em Energia REN - State Grid, S.A. \\ Rua Cidade de Goa, nº4-B - 2685-038 Lisboa, Portugal \\ (e-mail: rui.alves@ rdnester.com, francisco.reis@ rdnester.com) \\ ${ }^{2}$ China Electric Power Research Institute \\ (e-mail: chliang@epri.sgcc.com.cn)
}

\section{Introduction}

The Energy Roadmap 2050 issued by the European Commission establishes the decarbonisation of energy systems as a top priority, while ensuring security of energy supply and increasing competitiveness [1]. Largely motivated by these European Union's ambitious goals, the ongoing evolution of energy systems is demanding a massive integration of distributed renewable energy sources (DRES), along with a more active behavior of consumers in participating in demand side response (DSR) initiatives [2].

As this happens, the complexity of operational and planning activities of power systems is dramatically increasing [5], rising the need for a reinforced collaboration between TSOs and DSOs. Despite the interaction among grid operators being already a common practice at different levels, TSOs and DSOs must enlarge awareness on each other's grid as actions taken tend to have a greater impact on neighboring networks and need to be smartly coordinated.

Adopting a collaborative TSO-DSO approach requires significant amounts of additional information to be exchanged [3]. Enhancing the present framework of interaction between TSOs and DSOs by establishing efficient procedures to exchange data in a fast and reliable manner is crucial to increase system observability from both grid operators' standpoints. Moreover, improving levels of data exchange is the starting point for allowing grid operators to explore a shared use of flexibility resources to meet system needs in a perspective of keeping its high standards of efficiency, security of supply and quality of service.

High quality data exchange among TSOs and DSOs is

This is an Open Access article distributed in accordance with the Creative Commons Attribution Non Commercial (CC-BY-NC-ND 4.0) license, which permits others to copy or share the article, provided original work is properly cited and that this is not done for commercial purposes. Users may not remix, transform, or build upon the material and may not distribute the modified material (http://creativecommons.org/licenses/by-nc/4.0/) 
needed for several purposes. Those comprise the possibility of further optimizing operational and planning procedures which are normally not extensively coordinated between grid operators, such as: ancillary services; congestion management; voltage control; fault location; grid topology reconfiguration; system security assessments; maintenance scheduling; system planning and development; among many others.

In this paper, the need for improved data exchange between TSOs and DSOs is illustrated along with the major benefits and synergies emerging from a strengthened TSO-DSO collaboration. Examples, based on real case-studies, underline the positive impact of coordinating actions and improving information sharing among grid operators.

\section{Challenges and Framework of TSO-DSO Data Exchange}

Due to the present landscape of European energy systems, with TSOs and DSOs facing new and mutual challenges, an appropriate definition of the scope of data to be shared among grid operators is of most importance. Main variables such as the type of data to be exchanged, at which exchange rates and concerning which points of the distribution and transmission networks, are to be explicitly defined by both TSOs and DSOs to ensure the exchange of most adequate and meaningful data [6].

In this regard, future European Electricity Network Codes [7] will play an important role in supporting TSOs and DSOs defining the right levels of data to be exchanged between them.

\subsection{Grid Operators' Challenges: Correlated Need for Data Exchange}

Common challenges for both TSOs and DSOs include: maintaining proper levels of distribution and transmission grids' observability; enhance flexibility of renewable energy sources (RES) and leverage the deployment of demand side response [3]. For successfully meeting these challenges, different needs of data exchange between grid operators are emerging.

\section{I) Increase System Observability}

The growing integration of RES, mainly connected at the distribution level, and a lesser amount of large thermal generation units connected to transmission systems is likely to place TSOs facing a lack of overall system observability. In general, TSOs also do not have the desired observability levels of relevant assets of distribution infrastructure such as $\mathrm{HV}$ lines and substations. Future observability of energy systems, as a fundamental tool to allow TSOs to ensure system security, might be at risk if appropriate levels of TSODSO information exchange are not put in place.

From a DSO perspective, observability of transmission systems has always been very limited and, indeed, unnecessary. However, under a bidirectional power flow paradigm, increased observability of transmission systems is needed by DSOs. The exchange of data with TSOs allows DSOs to better manage their grids in a further efficient and secure way, namely in increasing penetration of DRES and in the ever more frequent process of reconfiguring the topology of distribution network.

\section{II) Increase RES Flexibility}

Reduced flexibility of distribution and transmission networks is a consequence of the existing limited degree of RES controllability. Even if restricted, available control capabilities of RES are becoming a more valuable resource to both TSOs and DSOs and must be exploited as efficiently as possible.

The exchange of data between grid operators is needed to operationalize an increased and shared use of flexibilities to support both TSOs and DSOs. Flexibility resources at the distribution level should be available to assist TSOs in operating transmission grid and vice-versa. Moreover, when implementing RES power control measures TSOs and DSOs must always act in coordination due to the possible impact of this type of actions into each other's grid [3].

\section{III) Deploy Demand Side Response}

Demand side response is one of the fastest developing areas in the energy sector. Nevertheless, even if the benefits of DSR are well recognized, the use of this type of flexibility is still far behind its full potential [4].

Although the majority of DSR potential capacity is connected at the distribution level, TSOs also play a fundamental role in enabling DSR. Grid operators at both transmission and distribution levels need to find a collaborative position on DSR to allow a complete exploitation of its benefits.

TSOs and DSOs will have to work together to unlock the potential of DSR and ensure its optimal usage to support their grids. In this process, grid operators will also need to enhance information exchange procedures, namely to improve awareness on the available capacities on each other networks to participate in such type of initiative. 


\subsection{Scope of TSO-DSO Data Exchange}

Having evidenced the need for improved TSO-DSO interaction, a clear definition of the scope of data to be exchanged is a demanding process. The large dimension of power networks requires limiting the amount of information to be shared between TSOs and DSOs due to technical feasibility reasons. Collaboration is necessary to define which data is relevant to be exchanged in accordance with the associated resulting benefits.

The priority in supporting grid operators on the process of defining the right extent of information exchange is also recognized by the European Electricity Network Code on Operational Security (NC-OS) [9]. It sets the future legally binding requirements of data to be exchanged between grid operators towards a better TSODSO interaction.

Defining the observability areas of both TSOs and DSOs is a necessary step to identify which points of the distribution and transmission grids will be considered in the process of exchanging information. From a TSO perspective, the observability area refers to its own transmission system and relevant parts of the distribution grid in which the TSO implements real-time monitoring, modelling or other type of information is exchanged with the neighboring DSO [10]. The same concept of observability area applies to the DSO, which concerns its own distribution system and relevant parts of the transmission network in which the DSO implements data monitoring and exchange with the TSO. Ideally, the exchange of data between grid operators should be more detailed and intense near the border between transmission and distribution networks, since it corresponds to the electrically closer area to the neighboring network where actions taken by TSOs or DSOs can severely impact the operation and planning activities of each other's grid.

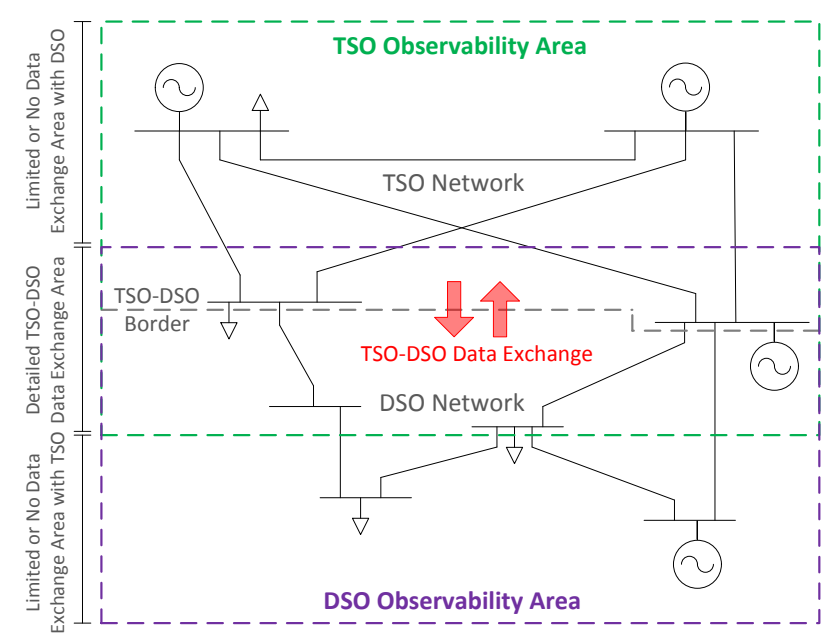

Fig 1 Concept of observability area - TSOs and DSOs' standpoints.
Different types of data to be exchanged between TSOs and DSOs, as proposed in the NC-OS, include three distinct categories:

A) Real-time Data - refers to the actual information from the network and its relevant users, necessary to perform real-time analysis of the transmission or distribution system [10].

B) Scheduled and Forecast Data - refers to the information from the network and its relevant users for day ahead and intra-day, used for operational security analysis of the transmission or distribution systems during this timeframes of operational planning [10].

C) Structural Data - refers to general information from the network and its relevant users, necessary for the models used to perform operational security analysis of the transmission and distribution systems in any timeframe, but also for system planning purposes [10].

\section{Areas for TSOs and DSOs Collaboration}

Benefits at both power systems' operation and planning levels can be leveraged through a closer interaction among grid operators, in particular in some of the following areas in which TSO-DSO data exchange is frequently limited:

\section{I) Ancillary Services}

As distributed resources of energy such as DRES, energy storage systems and DSR disseminate, the availability of flexible thermal generating units to provide ancillary services to the system might reveal insufficient. To avoid a shortage of this type of services, TSOs might look for viable alternatives to keep on meeting system's needs in terms of ancillary services.

The use of mentioned energy distributed resources is becoming a well-recognized solution to assist TSOs in providing a variety of system services. In this context, DSOs will need to be strongly engaged in the process of triggering this kind of services as the majority of the available distributed resources are connected to distribution networks. So, it becomes essential that TSOs and DSOs are able to articulate in order to optimally use flexibility resources (distributed generators, consumers and emerging service providers) for the provision of ancillary services.

\section{II) Congestion Management}

The further accommodation of intermittent generation into power networks is changing power flows direction and its path. As a consequence, congestion of distribution and transmission grids is expected to become a more 
frequent event.

In this sense, active power management will gain greater importance as a tool to allow both TSOs and DSOs to manage congestions on their systems. Different methods to perform active power management might include: active power control of generating units, including RES; management of energy storage devices; active demand management; control of different devices to manage active power such as FACTS or HVDC links.

A more structured cooperation among grid operators when dealing with congestions will help finding optimal solutions to overcome overloads in their networks. Again, the exchange of information between TSOs and DSOs is also relevant to ensure that actions taken by any of the grid operators are coordinated due to their possible crossborder impact.

\section{III) Voltage Control}

Preserving power systems' voltage levels within the desired ranges is usually ensured by TSOs through tap changing of reactor and capacitor devices. Frequently, DSOs also use this kind of devices to adjust reactive power levels injected into distribution networks ${ }^{1}$. The fact is that this type of actions, if not coordinated, may not only influence local voltage profiles, but, instead, impact voltage levels in the whole system.

In this perspective, TSO-DSO data exchange concerning reactor and capacitor devices available in both transmission and distribution networks is needed to allow TSOs and DSOs to anticipate any possible impact into their systems and coordinate actions to optimize use of reactive power control resources. Additional benefits emerge if DSO's devices are made available for TSO usage and vice-versa.

\section{IV) System Security}

Ensuring security of the system has always been a major responsibility of TSOs. Nevertheless, possibilities for collaboration with DSOs to enhance short and longterm system security are significant.

Operational security analyses performed by TSOs frequently do not have into consideration relevant parts of distribution networks, mainly due to lack of information concerning DSOs' grid. A joint assessment of security levels of both transmission and distribution systems would allow a more secure operation of the system and is only viable through effective data exchange.

${ }^{1}$ Typically, DSOs are driven by reducing system losses, while TSOs are driven by guaranteeing overall voltage stability.
Also, the involvement of DSOs in restoration plans can contribute to a more secure system. DSOs and TSOs must enhance collaboration in exploiting full potential of widespread DRES capabilities in order to manage system's restoration in a faster, better controlled and economic manner.

\section{V) System Planning and Development}

Because of the significant investments usually needed to expand transmission and distribution grids' infrastructure, planning and development activities of power networks need to be performed under economically efficient criteria. Thus, TSOs and DSOs must work together on integrated planning and development to promote efficiencies and cost savings in network infrastructure's development.

By improving collaboration on system planning, grid operators approach to expand the system should reflect the mutual impact that intended reinforcements might have in one another's transmission and distribution networks. TSOs and DSOs must improve interaction to intelligently invest on their grids and create synergies to avoid unnecessary investments and ensure that best planning and development decisions are jointly made to minimize overall system costs. 
Table 1 Summary of opportunities for enhancing TSO-DSO collaboration and associated benefits.

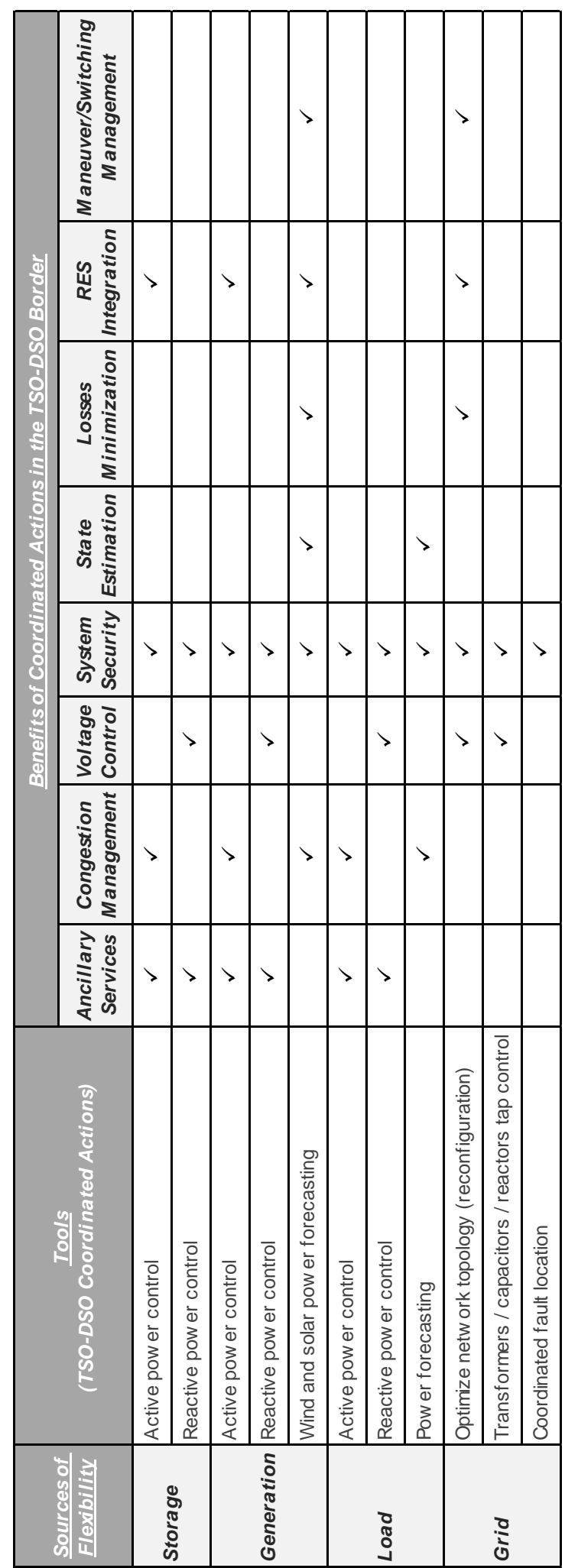

\section{Case-Studies}

Illustrative case-studies, based on real-world examples, intend to show how the current lack of TSODSO communication may jeopardize the operation of a power system under specific topological and operational network conditions. Recommendations given highlight the real possibility of mitigating existing problems by simply enhancing collaboration and data exchange between grid operators.

\section{I) Active Power Control Coordination to Manage Congestion}

This case presents a $400 \mathrm{kV} / 60 \mathrm{kV}$ TSO substation, located at the border between the transmission network $(400 \mathrm{kV})$ and the distribution grid (60 kV lines), to which a wind farm is connected at the $60 \mathrm{kV}$ side. In normal operating conditions the wind plant injects power into transmission network which then flows mainly through two $400 \mathrm{kV}$ lines.

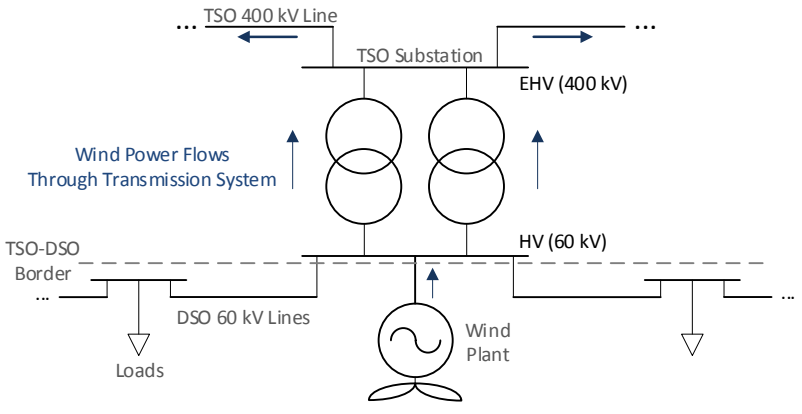

Fig 2 Wind power flows over $400 \mathrm{kV}$ lines - Normal operation state.

Whenever one of the $400 \mathrm{kV}$ lines goes under maintenance, the disconnection of the wind plant from the system is a common practice of the TSO. This is due to the existing possibility of DSO $60 \mathrm{kV}$ lines becoming overloaded in case a faulty condition occurs in the other $400 \mathrm{kV}$ line which will consequently also become out of service. This is an inefficient approach because, under the mentioned scenario, DSO lines usually have available capacity to withstand some of the wind power flows and a partial curtailment of wind power imposed by the TSO should be sufficient to avoid congestion problems and ensure maximization of RES integration. However, as the TSO lacks information concerning the distribution system and is not able estimate how much wind power should be curtailed, may opt for shutting down the entire plant.

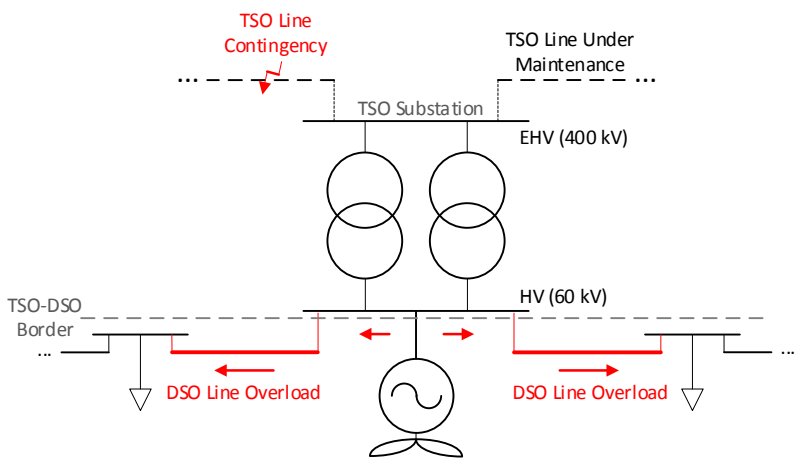

Fig 3 Wind power flows over $60 \mathrm{kV}$ lines - Overload of DSO lines. 
The solution for avoiding the disconnection of the wind farm from the system when a $400 \mathrm{kV}$ line is out of service and, instead, optimize wind power curtailment, consists on improving the collaboration between the TSO and DSO. In this particular example, more data should be accessible to the TSO, (e.g. for operational planning purposes), concerning the available capacities of distribution lines to allow the estimation of how much wind power can be injected into distribution grid. TSO and DSO should improve data exchange and coordinated actions to curtail wind power when a transmission line goes under maintenance.

\section{II) Voltage Control Through TSO-DSO Reactive Power Management}

Capacitor banks installed in transmission and distribution networks are used to control voltage levels. Fig 4 represents a situation in which capacitor bank $\mathrm{C}_{1}$ is managed by the TSO and connected to its substation (60 $\mathrm{kV}$ side), while $\mathrm{C}_{2}$ and $\mathrm{C}_{3}$ belong to the DSO and are linked to the MV distribution grid.

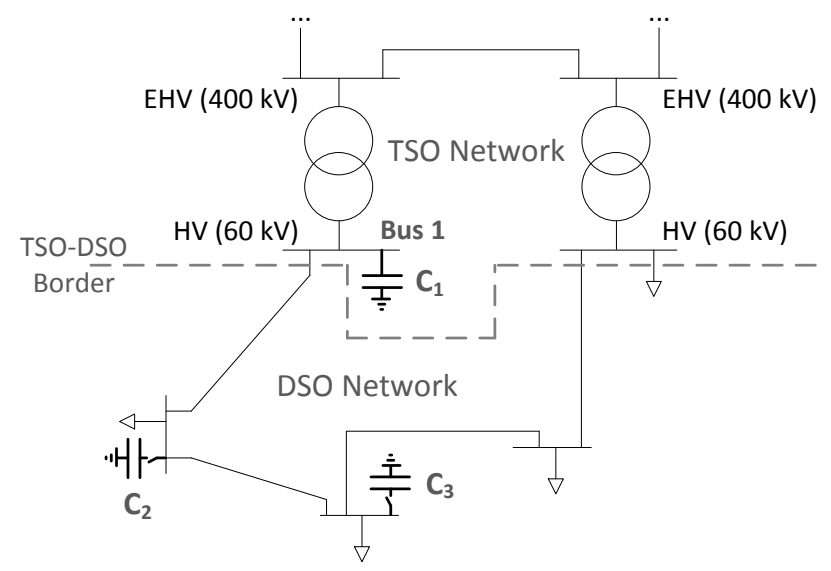

Fig 4 Reactive power management $\left(\mathrm{C}_{1}, \mathrm{C}_{2}, \mathrm{C}_{3}\right)$ -

TSO-DSO voltage control.

$\mathrm{C}_{1}$ is connected to the system at roughly half of its rated capacity in a normal state of operation, while DSO's capacitors $\mathrm{C}_{2}$ and $\mathrm{C}_{3}$ are usually disconnected from the system. Despite there is no TSO-DSO coordination in defining the state of operation of $\mathrm{C}_{1}, \mathrm{C}_{2}$ and $\mathrm{C}_{3}$, under the mentioned usual state of operation voltage levels are kept within desired range.

As capacitors are independently managed by the different grid operators, violation of the voltage limits at the $60 \mathrm{kV}$ busbar of the TSO substation (Bus 1) may occur when the DSO connects $\mathrm{C}_{2}$ and $\mathrm{C}_{3}$ to the system to meet a provisional local need of further reactive power injection into the distribution network, spreading the increase of voltage levels upwards. Without coordinating actions between TSO and DSO when managing reactive power, voltage problems will arise compromising the security of the system.

Therefore, in this particular case the TSO and DSO should collaborate in anticipation to optimize the state of operation of the capacitor banks in order to meet additional reactive power needs of the DSO without violating voltage limits at the TSO level.

\section{III) Increase Observability Area to Enhance System Security}

A scenario, where the observability of the TSO regarding the distribution system is very limited, is presented. Accordingly, when the TSO performs a $(\mathrm{N}-1)$ contingency analysis to evaluate the security of the system is only looking at the impact of contingencies on its own transmission network, neglecting its influence on the distribution grid.

Under regular operating conditions of the system, in which the distribution network is a non-observable area for the TSO, the TSO is able to successfully run a $(\mathrm{N}-1)$ contingency analysis and has no security reasons for changing the operational conditions of the system.

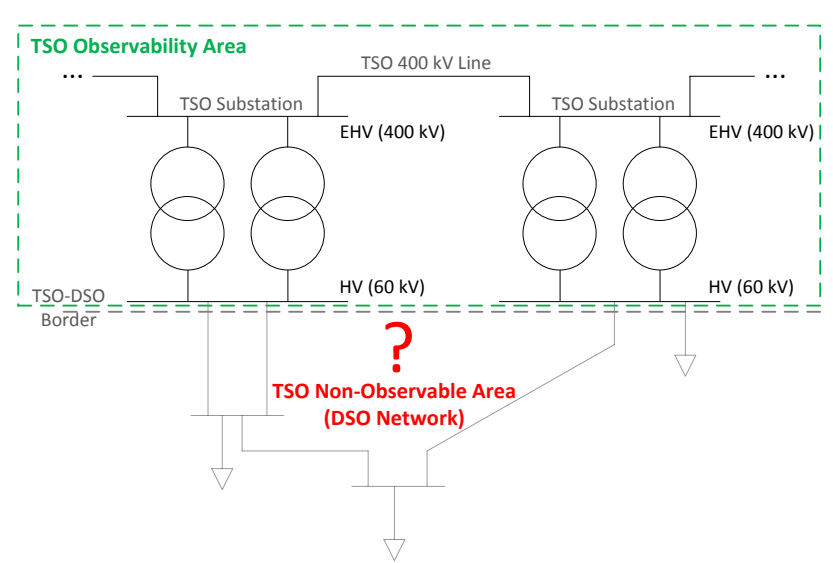

Fig 5 Limited TSO observability area - DSO network is a nonobservable area to the TSO.

Nonetheless, if a contingency occurs in the $400 \mathrm{kV}$ transmission line connecting the two TSO's substations, the DSO will face overload problems in one of its $60 \mathrm{kV}$ lines. Because the TSO does not jointly assess the security of the system considering both the transmission and relevant parts of the distribution networks, is not able to foresee such kind of problem and consequently does not adapt the operational conditions of the system to cope with the (N-1) system security criterion considering both grids. 


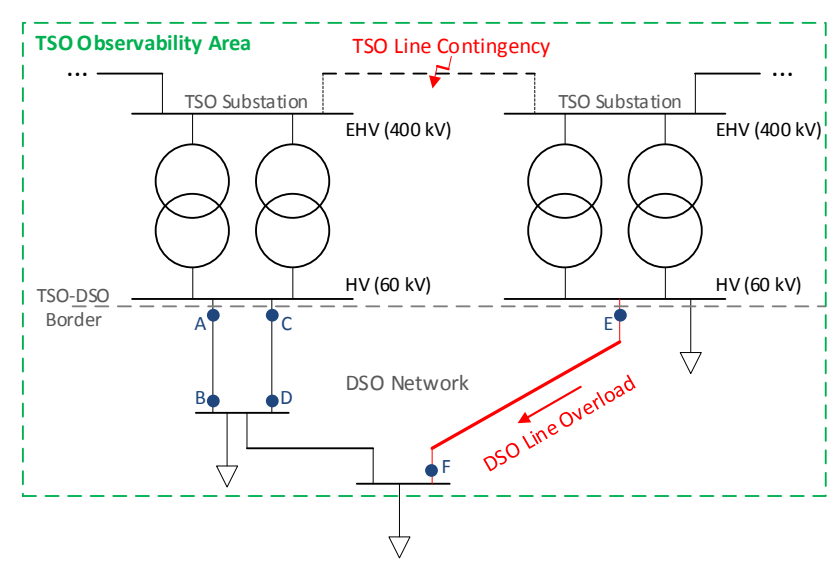

Fig 6 Enlarged TSO observability area - Joint security assessment of both transmission and distribution systems detects overload in DSO side. New observability points: A, B, C, D, E and F.

Enlarging the observability area of the TSO would allow a more secure operation of the system. Additional data exchange between the TSO and DSO (Table 2), concerning the distribution grid, is a vital requirement to ensure that the TSO has into consideration the possible impact of contingencies in relevant parts of the distribution network and is in condition to act accordingly to anticipate congestion scenarios on the distribution side.

Table 2 illustrates a set of additional data, based on network points marked on Fig 6 (A to F), that could be beneficial to share with the TSO to improve its observability on the distribution network:

Table 2 Sample of measurements for DSO data exchange with TSO to improve network observability.

\begin{tabular}{|c|c|c|c|c|}
\hline \multirow{2}{*}{ Lecation } & \multirow{2}{*}{$\begin{array}{l}\text { Network } \\
\text { Elements }\end{array}$} & \multicolumn{3}{|c|}{ Iyoe of Data Exchance } \\
\hline & & Real-Time & Scheduled & Structural \\
\hline DSO Network - Bays: & $60 \mathrm{kV}$ Bays: & & \multirow{4}{*}{$\mathrm{n} / \mathrm{a}^{*}$} & \multirow{4}{*}{$n / a^{*}$} \\
\hline A & Switch Breaker & Status (VO) & & \\
\hline c & \multirow{2}{*}{$\begin{array}{l}\text { Voltage Transformer } \\
\text { Current Transformer }\end{array}$} & $\mathrm{V}(\mathrm{rms})$ & & \\
\hline $\mathrm{E}$ & & $\mathrm{I}(\mathrm{rms})$ & & \\
\hline DSO Network - Lines: & \multirow{5}{*}{$60 \mathrm{kV}$ Lines } & \multirow{5}{*}{$n / a^{*}$} & \multirow{5}{*}{$\begin{array}{l}\text { Scheduled } \\
\text { Unavailability } \\
\text { Information }\end{array}$} & Resistance - R - $(\Omega)$ \\
\hline$A$ to $B$ & & & & Reactance - X - $(\Omega)$ \\
\hline$C$ to $D$ & & & & Susceptance - B - $\left(\Omega^{-1}\right)$ \\
\hline \multirow[t]{2}{*}{ E to $F$} & & & & Length $-\mathrm{L}-(\mathrm{km})$ \\
\hline & & & & $\begin{array}{c}\text { Seasonal Rated } \\
\text { Capacity- } S_{n}-(M V A)\end{array}$ \\
\hline
\end{tabular}

\section{Conclusions}

As TSOs and DSOs face complex challenges to plan and operate their systems, a better collaboration and data exchange between grid operators is a key approach to meet those challenges.

This paper has shown different areas where the present framework of TSO-DSO interaction can be enhanced based on an improved exchange of data and proper definition and application of the observability area.
Real case-studies unveiled the important role of TSODSO data exchange to cope with overload, voltage and security problems, which have confirmed the positive impact of collaboration in improving integration of RES and enhancing system security.

\section{References}

[1] E. Commission, "Energy Roadmap 2050”, Luxembourg: Publications Office of the European Union, 2012.

URL:https://ec.europa.eu/energy/sites/ener/files/docu ments/2012_energy_roadmap_2050_en_0.pdf

[2] evolvDSO, "Deliverable 1.1 - Definition of a Limited but Representative Number of Future Scenarios", 2014.URL:http://www.evolvdso.eu/getattachment/70 a9e337-5fb3-4300-a7d5-0b5b0b56ab1f/Deliverable1-2.aspx

[3] ENTSO-E, "Towards smarter grids: Developing TSO and DSO roles and interactions for the benefit of consumers", Brussels, Belgium, 2015.

URL:https://www.entsoe.eu/Documents/Publications /Position\%20papers\%20and\%20reports/150303_EN TSO-E_Position_Paper_TSO-DSO_interaction.pdf

[4] ENTSO-E, "Demand Side Response Policy Pape,", 2014.URL:https://www.entsoe.eu/Documents/Public ations/Position\%20papers\%20and\%20reports/14091 5_DSR_Policy_web.pdf

[5] N. Silva et al., "The Interaction Between DSO and TSO to Increase DG Penetration - The Portuguese Case”, in CIRED Workshop, Lisbon, 2012.

[6] evolvDSO, "Deliverable 1.3. - Preliminary assessment of the future roles of DSOs, future market architectures and regulatory frameworks for network integration of DRES", 2014.

URL:http://www.evolvdso.eu/getattachment/26ff93f 4-46d1-4e13-9e15-215bb3159c10/D1-3-v1-0.aspx

[7] ENTSO-E Network Codes Website [Online]. URL:http://networkcodes.entsoe.eu

[8] P. Djapic et al., "Taking and Active Approach", IEES Power and Energy Society Magazine, July/August 2007.

[9] ENSTO-E, "Network Code on Operational Security”, 2013.

URL:http://networkcodes.entsoe.eu/wpcontent/uploads/2015/01/130924-AS-

NC_OS_2nd_Edition_final.pdf 
[10]ENTSO-E, "Supporting Document for the Network Code on Operational Security”, 2013.

URL:http://networkcodes.entsoe.eu/wp-

content/uploads/2013/08/130924-AS-NC-

OS_Supporting_Document_2nd_Edition_final.pdf 